\title{
3D Reconstruction of Tree-Crown Based on the UAV Aerial Images
}

\author{
Chao Xu, ${ }^{1}$ Zeping Lu, ${ }^{1}$ Guangquan $\mathrm{Xu},{ }^{2}$ Zhiyong Feng, ${ }^{2}$ \\ Hongyan Tan, ${ }^{3}$ and Haifeng Zhang ${ }^{1,4}$ \\ ${ }^{1}$ School of Computer Software, Tianjin University, Tianjin 300072, China \\ ${ }^{2}$ School of Computer Science and Technology, Tianjin University, Tianjin 300072, China \\ ${ }^{3}$ High Performance Network Lab, Institute of Acoustics, Chinese Academy of Sciences, Beijing 100190, China \\ ${ }^{4}$ Space Star Technology Co., Ltd., Beijing 100086, China
}

Correspondence should be addressed to Guangquan Xu; xuguangquan@tju.edu.cn

Received 11 June 2015; Accepted 21 July 2015

Academic Editor: Krishnaiyan Thulasiraman

Copyright (c) 2015 Chao Xu et al. This is an open access article distributed under the Creative Commons Attribution License, which permits unrestricted use, distribution, and reproduction in any medium, provided the original work is properly cited.

\begin{abstract}
The algorithm for 3D reconstruction of tree-crown is presented with the UAV aerial images from a mountainous area in China. Considering the fact that the aerial images consist of little tree-crown texture and contour information, a feature area extraction method is proposed based on watershed segmentation, and the local area correlation coefficient is calculated to match the feature areas, in order to fully extract the characteristics that can reflect the structure of tree-crown. Then, the depth of feature points is calculated using the stereo vision theory. Finally, the L-system theory is applied to construct the 3D model of tree. The experiments are conducted with the tree-crown images from UAV aerial images manually. The experiment result showed that the method proposed in this paper can fully extract and match the feature points of tree-crown that can reconstruct the 3D model of the tree-crown correctly.
\end{abstract}

\section{Introduction}

In recent years, Unmanned Aerial Vehicle (UAV) imaging has become the irreplaceable mapping tool. We can get multiple imaging from multiangle and multiposition during a voyage of UAV [1]. Processing on the UAV sequential images with some related theories and technologies of 3D reconstruction, we can obtain the $3 \mathrm{D}$ models target on the ground. It is obvious that the height, color, texture, and outline information of the tree-crown from the images of high altitude is fuzzy. And the jitter, offset, and rotation of the aircraft can cause the motion and rotation of the camera. Furthermore, with the influence of many factors, the structure of the tree-crown has a lot of uncertainty, and it is difficult to establish empirical knowledge [2]. All these reasons make it very important theoretical guidance and practical significance to reconstruct the $3 \mathrm{D}$ model of treecrown from UAV aerial sequential images accurately.

In this paper, a tree-crown $3 \mathrm{D}$ reconstruction algorithm is proposed based on UAV aerial images, and our feature point extraction and matching method are fully considered the characteristics of the aerial images and the target to be reconstruction which can provide reference value to the future research. The modeling approach takes into account the structural characteristics of the canopy, and the full use of the existing information on more difficult issues as good canopy modeling is applied to achieve better results.

A brief review of relevant researches is presented in the second part. Then on the basis of the predecessors, the details of the algorithm are described in the third part, while in the fourth part, the experiments are demonstrated to validate the algorithm and to make a conclusion and discussion.

\section{Related Work}

In present, most researches of the 3D reconstruction are focused on the stereo vision principle to process two or more images from different angles of the same target and to reconstruct the 3D shape of the target. Sun and Bergerson [3], Liu et al. [4], and Hapca et al. [5] proposed related 
reconstruction method and models of tree object in this idea. With this idea in the $3 \mathrm{D}$ reconstruction of aerial images, the jitter and translation of UAV can be considered to improve the whole model and results. Compared with their researches concerning the backbone and structure information of tree, our research considered the detailed information of the treecrown contour and the internal texture.

Tan et al. [6] proposed a 3D tree reconstruction method to predict the obscured branches with the visible branches, and Tang et al. [7] proposed a 3D tree reconstruction method based on areas layered, so as to calculate the biological parameters of the tree. However, our method is concerned with the branches and also the characteristics of the outline. Cheng et al. [8] provided us with a good idea to determine the entire tree model with the help of determining the tree skeletons. There are many other researches on $3 \mathrm{D}$ tree reconstruction, such as [9-11], and their work gives important constructive advice to our work. Our research is more concerned with the unique features of the target tree-crown.

Although most of the researches are concerned with the backbone and structure information of tree, we consider the detailed information of the tree-crown contour and internal texture. It is very important to extract and to match the feature points of the target for $3 \mathrm{D}$ reconstruction. In our model and method, process is designed and conducted to fully extract the points that can reflect the structural characteristics of the target and to correctly match the feature points which directly affect the accuracy of the final results of $3 \mathrm{D}$ reconstruction. Commonly used methods of corresponding points matching can be classified as feature-based and region correlation coefficient based on matching method [1].

SIFT and SURF are typical matching methods based on typical features; for example, Hou et al. [12], Ciobanu and Côrte-Real [13], and Bellavia et al. [14] all used SIFT algorithm to extract feature points and to match them in $3 \mathrm{D}$ problems so as to achieve good results of the robustness and stability. Such algorithms have good resilience, stability and robustness for image rotation, zooming, and other changes, but these algorithms can only extract limited points when there are images with limited texture information.

Many scholars extract and match feature points based on region correlation coefficient; for instance, Wei et al. [15] put forward a method based on region correlation coefficient of gray image to match the feature points. Region-based correlation method is performed well in accuracy, but the calculate time is long. Further improvement is conducted; for example, Yang [16], Mori and Kashino [17], and Zhang et al. [18] have put forward some improvements on template matching method based on normalized correlation coefficient (NCC). These methods do not need complex preprocessing on images, and they only use the gray statistics of image itself [19]. Based on the analysis above, we select the appropriate feature extraction methods and efficient scanning methods to save up the time consumption.

In addition, considering the complex structure of the tree, 3D modeling approaches are commonly based on grid, since the canopy structure characteristics of aerial images are limited. Li [20] and Shlyakhter et al. [21] used Lindenmayer system (L-system) to automatically generate models of tree, which was applied to build complex models of trees in our proposed method.

On the basis of the current researches, a tree-crown $3 \mathrm{D}$ reconstruction algorithm is proposed. Firstly, feature points are extracted based on the watershed to match features by calculating the local area correlation coefficient (LACC) in the RGB color space; secondly, the depth information is achieved based on the principle of binocular stereo vision; finally, L-system is applied to the 3D modeling of the treecrown.

\section{3D Reconstruction Algorithm}

The flow of the proposed algorithm in this paper is presented in Figure 1, which is divided into six parts including image acquisition, preprocessing, feature extraction and matching, camera parameter estimation, depth calculation, and $3 \mathrm{D}$ modeling.

To ignore the impact from other parts for the $3 \mathrm{D}$ reconstruction, we intercept the same size of $128 * 128$ as the crown area of two successive aerial images manually, and GrabCut and BgCut [22] algorithm are applied to remove the background from tree-crown, leaving only the crown area.

3.1. Feature Extraction and Matching. Feature extraction and matching is a crucial step in $3 \mathrm{D}$ reconstruction, and the accuracy of matching feature points affects the final results directly. Due to the complexity of UAV aerial images, it is difficult to match the feature points as the increased point extractions.

Based on the analysis on the tree-crown image cut from the aerial images, we found that UAV aerial images contain scarce information of a single tree relatively, and the texture information of a tree-crown is similar, or nearly no texture, while the same area of the two image has great relevance. In response to these findings, a feature point extraction and matching method is designed with four parts as: feature extraction based on watershed; region matching based on LACC; elimination of the fault matching; mean geometric registration error.

3.1.1. Feature Extraction Based on Watershed. Since the two images were captured during a short interval, the illumination changes are small between the two images. And in the tree-crown images, area at the top of the trunk is relatively bright while the bottom area is relatively dark. So we use the watershed segmentation method to separate the light and dark areas in order to further extract the feature points that reflect the trunk structure of the tree $3 \mathrm{D}$ reconstruction.

Watershed segmentation algorithm usually takes gradient image as input, which can get the closed areas quickly. However, there is often oversegmentation phenomenon [19]. In this paper, the oversegmentation of watershed segmentation algorithm is just suitable to our requirements of fully separating the light and dark areas of the tree-crown.

The classic watershed segmentation algorithm is proposed by Vincent and Soille [23], and the algorithm is 


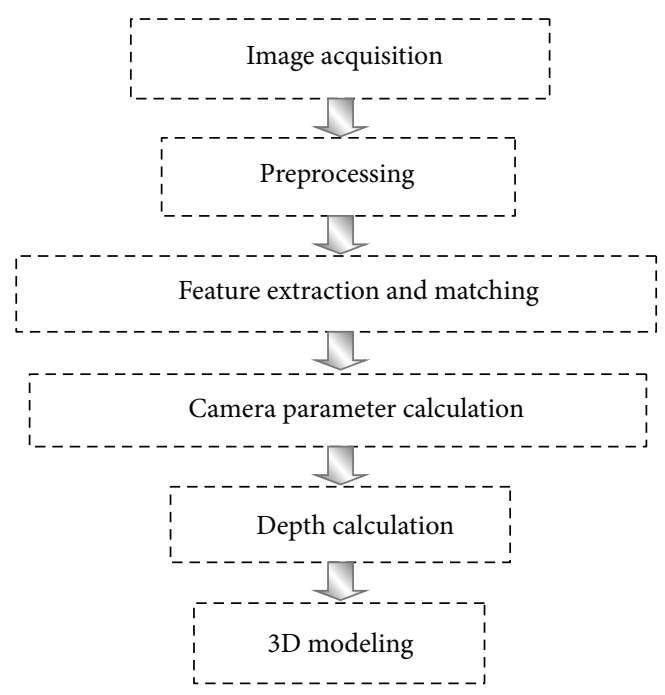

Figure 1: The flow of 3D reconstruction.

calculated through two steps: the first one is the sorting process and the other one is the submerging process. First of all, the gray-level height for each pixel is sorted from low to high. And then the process of submerging is achieved from low to high. For each local minimum value in the influent domain of h-order height, the first in first out (FIFO) structure is applied to archive the judgment and to complete the labeling process.

The watershed algorithm transforms the input image to the basin image. Then the boundary of the basin is the watershed. The gradient image is calculated as the input, and the gradient value is calculated as shown in (1); wherein, the $f(x, y)$ is the original image, and the $\operatorname{grad}(\cdot)$ is the gradient function:

$$
\begin{aligned}
& g(x, y)=\operatorname{grad}(f(x, y)) \\
& =\sqrt{[f(x, y)-f(x-1, y)]^{2}+[f(x, y)-f(x, y-1)]^{2}} .
\end{aligned}
$$

The watershed segmentation method used in this paper is represented as follows:

(i) Do gradient computation on the longitudinal direction with the Sobel operator. And the horizontal and vertical Sobel operator template are shown in (2), wherein $A$ is the original image:

$$
\begin{aligned}
& G_{x}=\left(\begin{array}{ccc}
-1 & 0 & 1 \\
-2 & 0 & 2 \\
-1 & 0 & 1
\end{array}\right) \times A, \\
& G_{y}=\left(\begin{array}{ccc}
1 & 2 & 1 \\
0 & 0 & 0 \\
-1 & -2 & -1
\end{array}\right) \times A .
\end{aligned}
$$

(ii) Smooth the gradient image by making opening and closing operations as shown in

open $($ src, element $)=\operatorname{dilate}(\operatorname{erode}($ src, element $))$,

close $($ src, element $)=$ erode $($ dilate $($ src, element $))$.

(iii) Segment the smoothed images using the watershed segmentation algorithm as shown in

$$
g(x, y)=\max \left(\operatorname{grad}(f(x, y)), g_{\theta}\right) .
$$

3.1.2. Region Matching Based on LACC. As mentioned above, the region matching can be achieved based on the LACC values which can be calculated in the RGB color space; please see Figure 2.

The LACC in the RGB color space is calculated in

$$
c_{i j}=R c_{i j}+G c_{i j}+B c_{i j} \text {, }
$$

wherein, $R c_{i j}, G c_{i j}$, and $B c_{i j}$ represent the three components $R, G$, and $B$, respectively. And we calculate them in (6) with $R$ as an example:

$$
\begin{aligned}
R c_{i j} & =\frac{\sum_{k=-w n}^{w n} \sum_{k=-w m}^{w m}\left[R_{1}\left(u_{i}^{(I)}+k, v_{i}^{(I)}+l\right)-\overline{R_{1}}\left(u_{i}^{(I)}, v_{i}^{(I)}\right)\right]\left[R_{2}\left(u_{j}^{(I I)}+k, v_{j}^{(I I)}+l\right)-\overline{R_{2}}\left(u_{j}^{(I I)}, v_{j}^{(I I)}\right)\right]}{n \sqrt{\delta_{i}^{2}\left(R_{1}\right) \times \delta_{j}^{2}\left(R_{2}\right)}}, \\
\bar{R}(u, v) & =\frac{1}{n} \sum_{k=-w n}^{w n} \sum_{k=-w m}^{w m} R_{1}(u+k, v+l)
\end{aligned}
$$

wherein, $R_{1}\left(u_{i}^{(I)}, v_{i}^{(I)}\right)$ and $R_{2}\left(u_{j}^{(I I)}, v_{j}^{(I I)}\right)$ represent the value of $R$ from two images, while $w n$ and $w m$ represent the half width and the half length of the sliding window. And we take the size of the rectangle circumscribed for the watershed area as the size of the sliding window. $\bar{R}(u, v)$ represents the mean value of $R$ for all the pixels in the sliding window, which can be represented for the standard deviation within a sliding window as shown in

$$
\delta(R)=\sqrt{\frac{1}{n} \sum_{k=-w n}^{w n} \sum_{k=-w m}^{w m}\left(R^{2}(u+k, v+l)-\bar{R}^{2}(u, v)\right) .}
$$




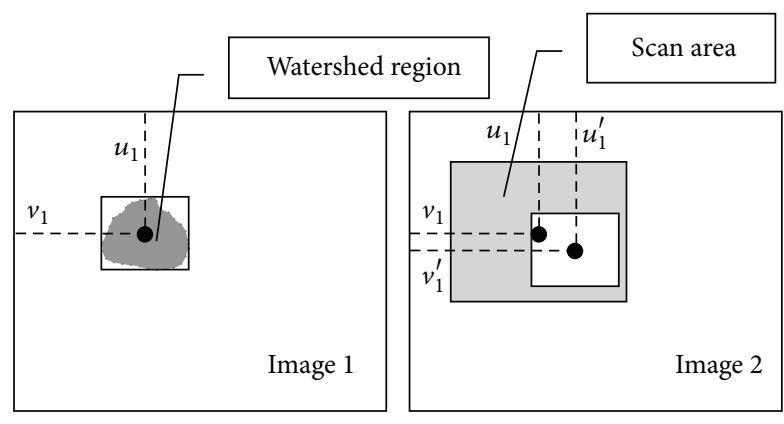

FIGURE 2: Region matching based on LACC.

The values of $R c_{i j}, G c_{i j}$, and $B c_{i j}$ are ranged in $(-1,1)$. If the current areas in the sliding windows from the two images are matched, the value correlation coefficient is closed to 1 .

The ultimate target of this procedure is to get the matched feature points for the two images. In this paper, we calculate the centroid values of the matched areas as the matching points, and their coordinates are calculated in

$$
\begin{gathered}
\overline{u_{i}}=\frac{1}{n_{i}} \sum_{k=0}^{n_{i}} u_{k}, \\
\overline{v_{i}}=\frac{1}{n_{i}} \sum_{k=0}^{n_{i}} v_{k} .
\end{gathered}
$$

In order to reduce the time consumption, the image disparity constraints of two images are taken into account to restrict the searching area. During the matching process, the sliding window is moved only in this area, not in the whole image area.

3.1.3. Eliminate the Fault Matching. In the matching procedure, only the local information of the area is considered. So we get the correct matching areas of the two images as well as some fault matched areas. In order to improve the results, we need to eliminate the fault matching results.

In this paper, we calculate the ratio of the biggest correlation coefficient value and the second coefficient value to eliminate the fault matching. According to paper [24], we find that the fault matching areas have many other matched areas with the similar correlation coefficient value. And then the ratio value will be bigger than the correct matched ones. So we set a threshold as a criterion to eliminate the matching process which has a ratio value higher than the threshold.

3.1.4. Mean Geometric Registration Error. Points from the two images can have a mapping relation as in

$$
X_{2}=H X_{1} \text {, }
$$

wherein $H$ is the homograph matrix of the two images and $X_{1}$ and $X_{2}$ represent the points from the two images.

We calculate the mean geometric registration error according to the principle of homograph as shown in Figure 3 and

$$
E n=\frac{1}{2 n} \sum_{i \in n}\left(\operatorname{dist}\left(x_{i}^{\prime}, H x_{i}\right)+\operatorname{dist}\left(x_{i}, H^{-1} x_{i}^{\prime}\right)\right),
$$

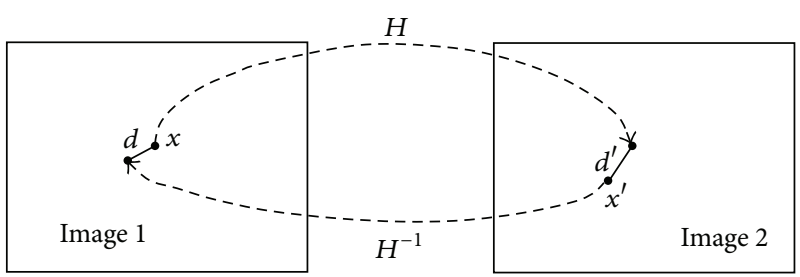

FIgURE 3: The principle of mean geometric registration error.

wherein, $\operatorname{dist}(\cdot)$ represents the distance between the two points $x_{i}$ and $x_{i}^{\prime}$.

Thus, if the points are matched correctly, the distance between $d$ and $d$ ' will be small, and it will result in a small $E n$. In other words, the value of En decreases when the veracity of the matching increases.

3.2. Depth Calculation. In this paper, the whole camera model is applied. Assume there is a $3 \mathrm{D}$ point $X=(X, Y, Z)^{T}$ in this projection model, and the correspondent point $x=$ $(x, y)^{T}$ is in the image; the two points $X$ and $x$ have the relationship as shown in

$$
\widetilde{x}=\frac{1}{\lambda} P \widetilde{X}=\frac{1}{\lambda} K(R \mid t) \widetilde{X}
$$

where $\tilde{x}$ and $\widetilde{X}$ represent the homogeneous coordinates of $x$ and $X ; \lambda$ is the scale factor between the two sides of the homogeneous vector equation, also called the projective depth; $K$ is the intrinsic parameter matrix; and $R$ is the rotation matrix and $t$ is the translation matrix.

We calculate the projection equation of the two treecrown images in (12); and the equation is solved to get the world coordinates of the point in (13):

$$
\begin{aligned}
\lambda_{1}\left(u_{1}, v_{1}, 1\right)^{T} & =K\left(R_{1} \mid t_{1}\right)\left(X_{W}, Y_{W}, Z_{W}, 1\right)^{T} \\
\lambda_{2}\left(u_{2}, v_{2}, 1\right)^{T} & =K\left(R_{2} \mid t_{2}\right)\left(X_{W}, Y_{W}, Z_{W}, 1\right)^{T}, \\
X_{W} & =-\frac{f_{v} T_{y}\left(u_{1}-u_{0}\right)}{f_{u}\left(v_{1}-v_{2}\right)} \\
Y_{W} & =-\frac{T_{y}\left(v_{1}-v_{0}\right)}{v_{1}-v_{2}} \\
Z_{W} & =-\frac{f_{v} T_{y}}{v_{1}-v_{2}} .
\end{aligned}
$$

The camera intrinsic parameter is the matrix

$$
K=\left(\begin{array}{ccc}
f_{u} & 0 & u_{0} \\
0 & f_{v} & v_{0} \\
0 & 0 & 1
\end{array}\right)
$$


which ignores the small rotation of the camera. Thus, we have the rotation matrix as

$$
R_{1}=R_{2}=\left(\begin{array}{lll}
1 & 0 & 0 \\
0 & 1 & 0 \\
0 & 0 & 1
\end{array}\right) .
$$

We assume that the translation vectors are of image $1 T_{1}=$ $\left(\begin{array}{lll}0 & 0 & 0\end{array}\right)$ and image $2 T_{1}=\left(\begin{array}{lll}T_{x} & T_{y} & 0\end{array}\right)$. There are some methods to estimate the parameters of the camera. With these known parameters, we can get the $3 \mathrm{D}$ coordinates of the feature points.

3.3. 3D Modeling. L-system is proposed by biologist Lindenmayer [25]. L-system can be later developed into an effective computer graphics simulation of nature scenery, and it is a language prompting system which controls parameters of certain symbols and words. L-system's right-hand Cartesian coordinate system is defined in Figure 4, which is composed of three directions defined by three vectors as $H, L$, and $U$ [17]. Commonly used commands of L-system include $3 \mathrm{D}$ positioning commands, special positioning commands, mobile commands, structure commands, and increasing or decreasing commands.

After the 3D points are configured through the method described above, we apply a specific method to get the trunk information of the tree-crown according to the points, and then we use $L$-system command to construct the trunk structure and further the whole tree with leaves. According to Figure 5, the method we used to get the trunk structure is described as follows:

(i) Find the highest point and the lowest point in the $Z$ coordinate and get the difference $d z$ as the tree height.

(ii) Select the highest point on the vertical axis as the center trunk of the tree, and set its depth as 1 . Add the trunk to TrunkList collection, and add the highest point to trunk points set IncludePoints.

(iii) Scan the feature points which are not in the collection IncludePoints to find the point with a minimum distance to the trunks in the collection TrunkList, and add the point into the collection IncludePoints.

(iv) Create a new trunk from the new point to its nearest trunk with 30 degrees. The depth of the new trunk plus one (the maximum depth of the tree is restricted to less than 4), and add the new trunk into the collection TrunkList.

(v) Go back to Step (iii) until all the points are included in the collection IncludePoints.

\section{Experiment}

Based on the above algorithm, two successive images are obtained during a voyage of UAV, which is manually cut for the tree-crown image with the same size as $128 * 128$ as shown in Figure 6(a).

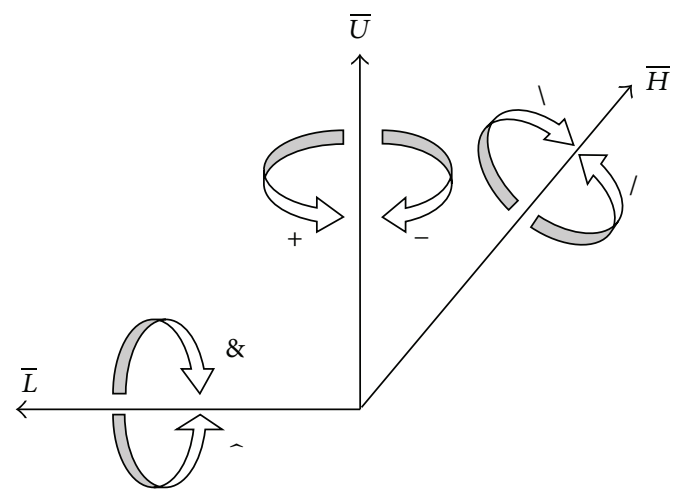

FIgURE 4: The coordinate system of L-system.

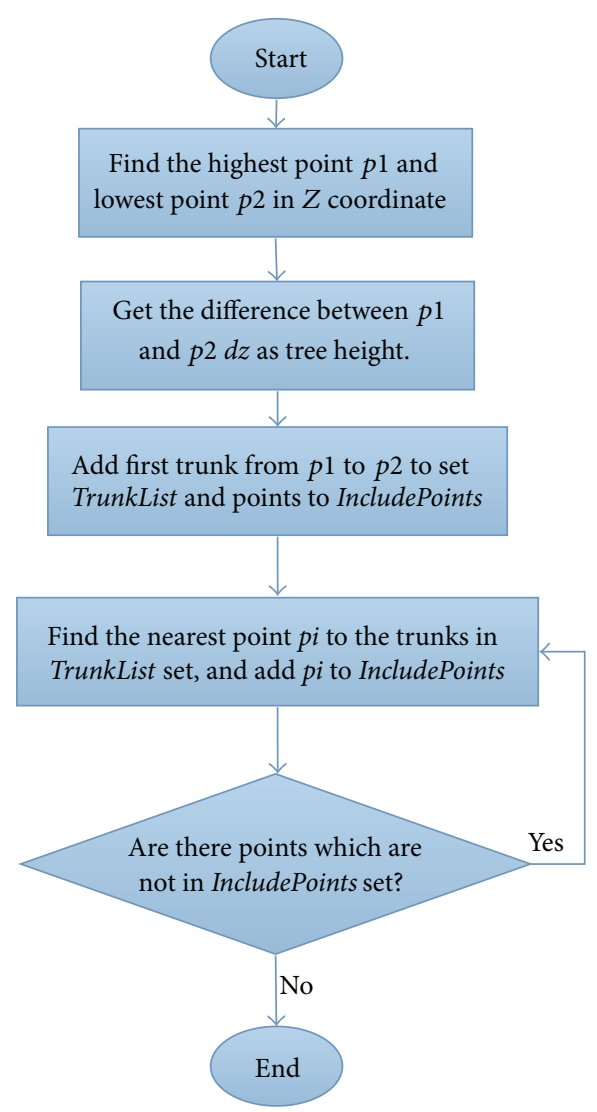

FIgURE 5: The construction process of the trunk structure.

In order to avoid the impact of noise outside the canopy region, we use BgCut [22] algorithm to remove image background, as shown in Figure 6(b).

For the next step, the light areas and dark areas of the tree-crown are separated on image 1 with the watershed segmentation algorithm. After obtaining the light and dark areas of the tree-crown, the numbers of pixels of each area are recorded, and the centroid of the areas is calculated as the feature points of image 1. In Figure 7, we can extract more bright points and dark points for the tree-crown, which can reflect the trunk structure of the tree. 


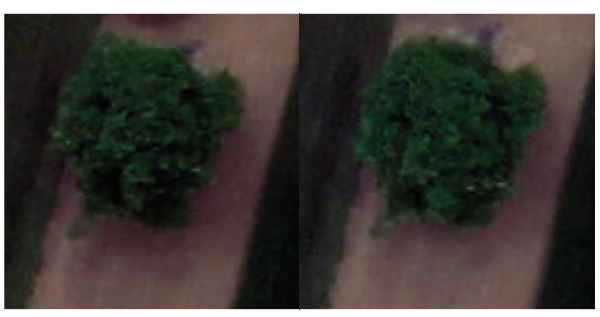

(a)

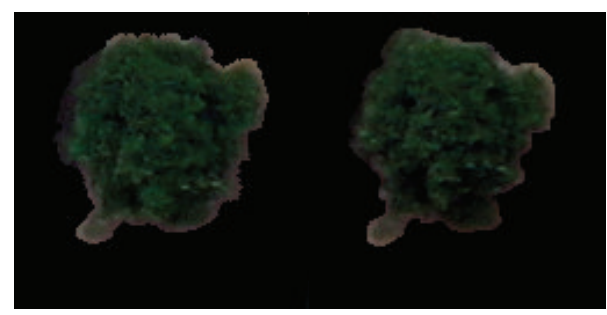

(b)

Figure 6: The two tree-crown images (a) with wackground; (b) without background.

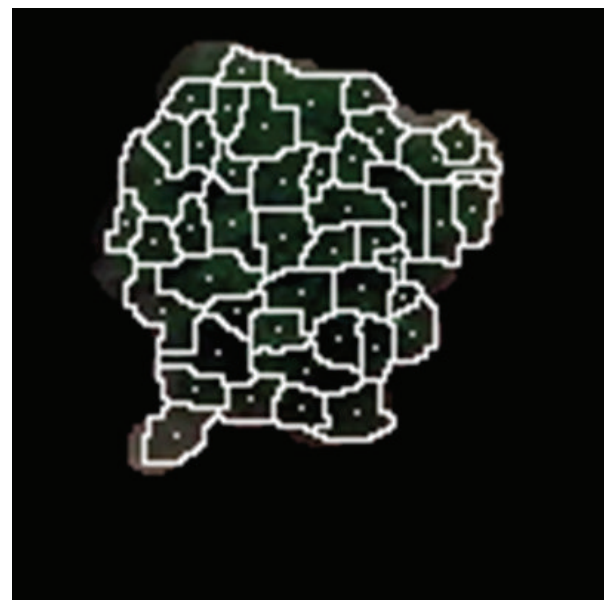

FIGURE 7: The result of watershed on tree image 1 and the center of the watershed areas.

Image 1 is separated into 47 areas with 47 feature points. Then the local area correlation coefficient is calculated in the RGB color space so as to find the matched area from image 2. The rough matching result is shown in Figure 8.

The points of image 2 are roughly matched with points of image 1 including some fault matching inevitably. So we set different thresholds described in Section 3 to find proper results to reconstruct the tree model.

The average geometric registration errors are calculated between the matched points, and the results are shown in Table 1 . We can see from the table, compared with the method proposed in paper [24], that the method we proposed can pick more feature points which are enough to construct the tree model, and these points can be matched more accurately. To take the appropriate threshold value $K$, a better reconstruction result can be achieved.

The trunk structure information of the tree-crown is calculated according to the method described in the above section, and the feature point set and the correspondence trunk structure of the tree with different threshold values are shown in Figure 9.

As seen from the results in Figure 9, if the threshold value is taken too large, there are still some false matching points, then the remaining error will affect the $3 \mathrm{D}$ modeling results, and if the threshold value is taken too small, there are some correct matching points which are removed, which results
TABLE 1: Mean geometric registration error.

\begin{tabular}{lcc}
\hline Method & $E n$ & N_Points \\
\hline SIFT-KNN [24] & 1047.997 & 5 \\
Our method with $(K \leq 1)$ & 1041.99 & 47 \\
Our method with $(K \leq 0.8)$ & 1040.63 & 14 \\
Our method with $(K \leq 0.6)$ & 1039.91 & 9 \\
Our method with $(K \leq 0.4)$ & 1038.23 & 4 \\
\hline
\end{tabular}

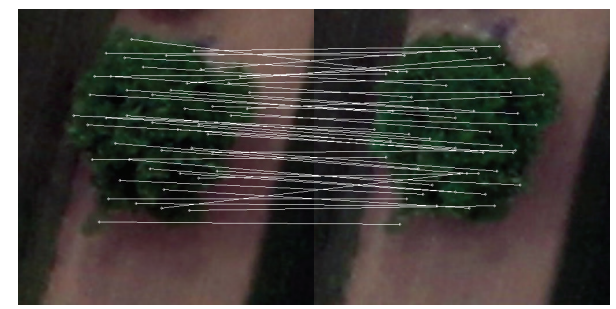

FIGURE 8: The matched points on the two images.

in the loss of information. So we use the threshold of 0.91 , and the result is shown in Figure 10. Finally, the leaves of the skeleton model can be reconstructed for the 3D model of the target tree.

\section{Conclusion}

UAV imaging has great practical significance in forestry and land planning. However, two-dimensional images lack the necessary depth information, which will be a limitation of the UAV aerial applications. In this paper, 3D reconstruction of vision technology is proposed to obtain the depth information from the 2D images of the object with great advantages. The $3 \mathrm{D}$ reconstruction based on UAV aerial image can be used in many practical applications.

In this paper, feature extraction and matching methods are proposed based on watershed segmentation algorithm, and local area correlation coefficient is introduced in the RGB color space, which can fully extract the feature points of the images so as to reflect the structure of the target treecrown. And reconstruction of the $3 \mathrm{D}$ model of the treecrown is designed based on the principle of stereoscopic vision from the limited information of the aerial images. In the proposed method in this paper, we make an effort to the camera imaging model of UAV. The method can apply to 

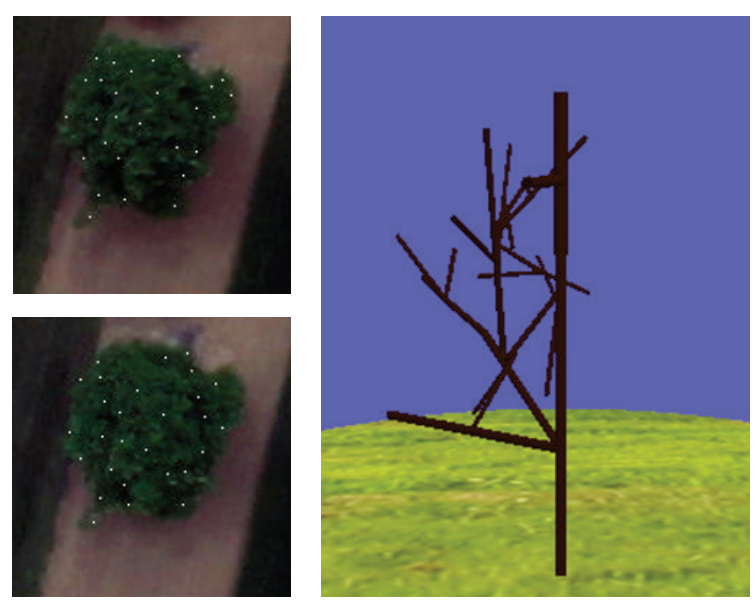

(a) Threshold 0.93
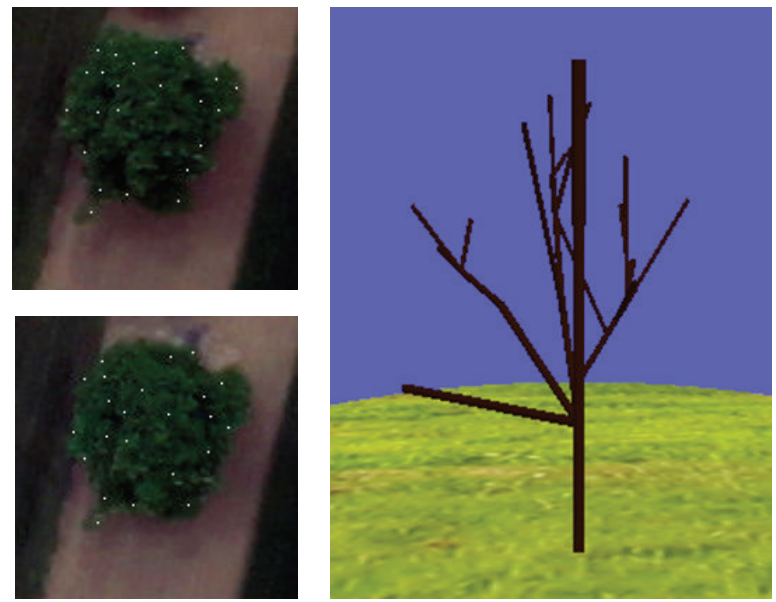

(c) Threshold 0.91
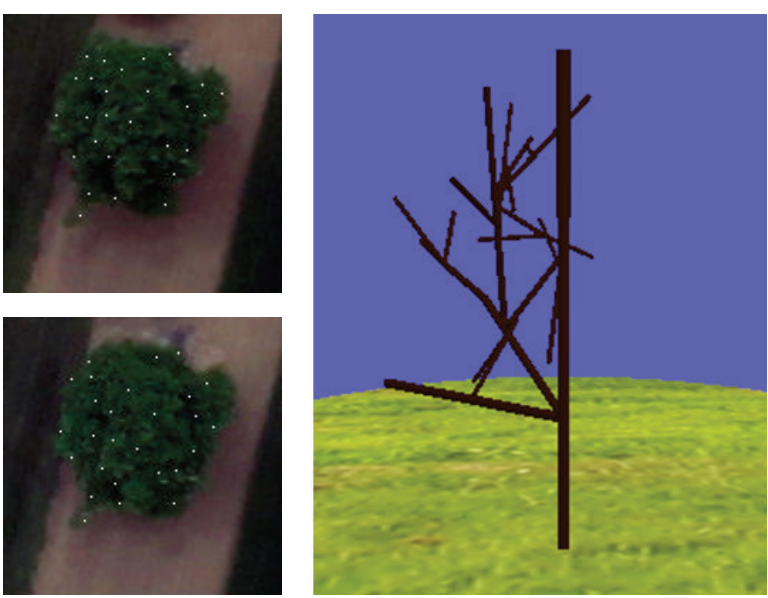

(b) Threshold 0.92
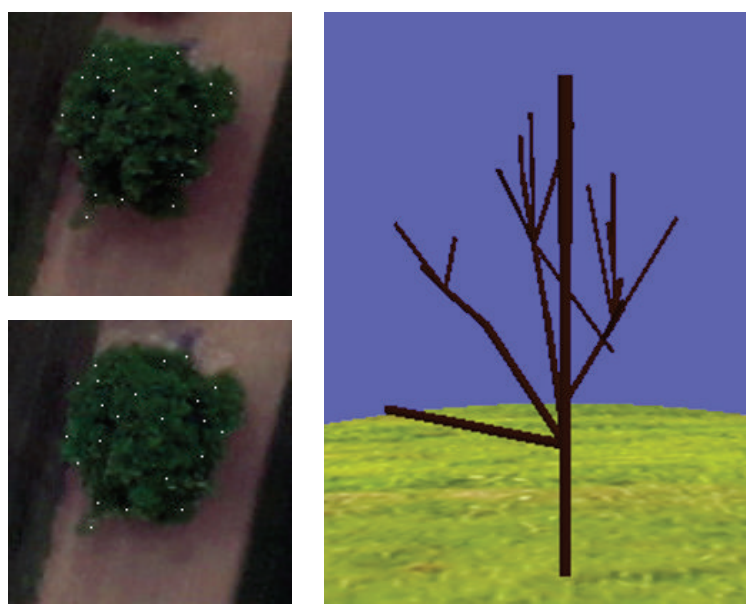

(d) Threshold 0.90

Figure 9: The result with different threshold; (a) with threshold as 0.93, 28 feature points are selected, and the result is poor because of the remaining disturbance; (b) with the threshold as 0.92, 27 feature points are selected, and it has a similar result as 0.93; (c) with the threshold as 0.91, 23 feature points are selected, and the result has an effective improvement; (d) with the threshold as 0.90 , only one feature point is lost against the result as 0.91 .

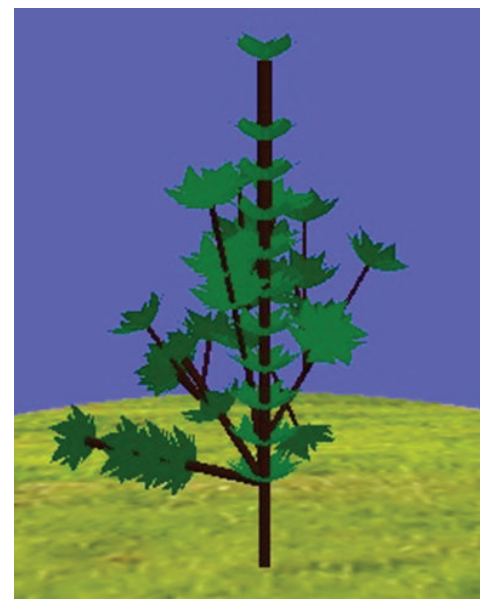

(a)
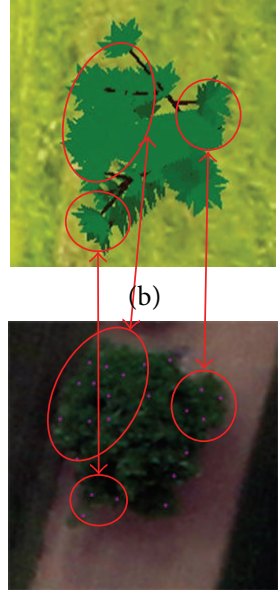

(c)

Figure 10: 3D model of the target tree. (a) The side view of the tree model; (b) the top view of the 3D tree model; (c) the original treecrown image.

the case of aerial images taken within a small interval, and the $3 \mathrm{D}$ reconstruction method has applicability for tree-crown.

\section{Conflict of Interests}

The authors declare that there is no conflict of interests regarding the publication of this paper.

\section{Acknowledgment}

This work is partially supported by National Natural Science Foundation of China (no. 61304262).

\section{References}

[1] L.-C. Li, Reconstruction of Terrain Based on Unmanned Aerial Vehicle Sequential Images and Its Application in the Navigation Research, National University of Defense Technology, Changsha, China, 2009. 
[2] C. Zhuo, M. Hong-Chao, and W. Jian-Wei, "3D tree modeling method based on airborne LiDAR data," Computer Engineering, vol. 38, no. 4, pp. 1-3, 2012.

[3] Y. Sun and E. Bergerson, "Automated 3D reconstruction of tree-like structures from two orthogonal views," in Proceedings of the International Conference on Acoustics Speech and Signal Processing, pp. 1296-1299, New York, NY, USA, 1988.

[4] Y.-H. Liu, H.-B. Wang, and W. Du, "3D reconstruction of treelike object based on images," Chinese Journal of Computers, vol. 25, no. 9, pp. 930-935, 2002.

[5] A. I. Hapca, F. Mothe, and J.-M. Leban, "A digital photographic method for 3D reconstruction of standing tree shape," Annals of Forest Science, vol. 64, no. 6, pp. 631-637, 2007.

[6] P. Tan, G. Zeng, J. Wang, S. B. Kang, and L. Quan, "Image-based tree modeling," ACM Transactions on Graphics, vol. 26, no. 3, article 87, 7 pages, 2007.

[7] S. Tang, P. Dong, and B. P. Buckles, “Three-dimensional surface reconstruction of tree canopy from lidar point clouds using a region-based level set method," International Journal of Remote Sensing, vol. 34, no. 4, pp. 1373-1385, 2013.

[8] Z.-L. Cheng, X.-P. Zhang, and B.-Q. Chen, "Simple reconstruction of tree branches from a single range image," Journal of Computer Science and Technology, vol. 22, no. 6, pp. 846-858, 2007.

[9] Y. Li, J. L. Gutiérrez-Chico, N. R. Holm et al., "Impact of side branch modeling on computation of endothelial shear stress in coronary artery disease: coronary tree reconstruction by fusion of 3D angiography and OCT," Journal of the American College of Cardiology, vol. 66, no. 2, pp. 125-135, 2015.

[10] P. J. Zarco-Tejada, R. Diaz-Varela, V. Angileri, and P. Loudjani, "Tree height quantification using very high resolution imagery acquired from an unmanned aerial vehicle (UAV) and automatic 3D photo-reconstruction methods," European Journal of Agronomy, vol. 55, pp. 89-99, 2014.

[11] F. Rottensteiner, G. Sohn, M. Gerke, J. D. Wegner, U. Breitkopf, and J. Jung, "Results of the ISPRS benchmark on urban object detection and 3D building reconstruction," ISPRS Journal of Photogrammetry and Remote Sensing, vol. 93, pp. 256-271, 2014.

[12] X.-D. Hou, Y.-F. Dong, H.-J. Guo, and X. Yang, "The method of pavement image splicing based on SIFT algorithm," in Proceedings of the WRI Global Congress on Intelligent Systems (GCIS '09), vol. 4, pp. 538-542, IEEE, Xiamen, China, May 2009.

[13] L. Ciobanu and L. Côrte-Real, "Iterative filtering of SIFT keypoint matches for multi-view registration in Distributed Video Coding," Multimedia Tools and Applications, vol. 55, no. 3, pp. 557-578, 2011.

[14] F. Bellavia, D. Tegolo, and E. Trucco, "Improving SIFT-based descriptors stability to rotations," in Proceedings of the 20th International Conference on Pattern Recognition (ICPR '10), pp. 3460-3463, Istanbul, Turkey, August 2010.

[15] L. Wei, S. Jin, and C. Wengang, "Image mosaic technology based on overlapped area linear transition method," in Proceedings of the 2nd International Congress on Image and Signal Processing (CISP '09), pp. 1-3, Tianjin, China, October 2009.

[16] Z. Yang, "Fast template matching based on normalized cross correlation with centroid bounding," in Proceedings of the International Conference on Measuring Technology and Mechatronics Automation, pp. 224-227, Changsha, China, March 2010.

[17] M. Mori and K. Kashino, "Fast template matching based on normalized cross correlation using adaptive block partitioning and initial threshold estimation," in Proceedings of the IEEE
International Symposium on Multimedia (ISM '10), pp. 196-203, Taichung, China, December 2010.

[18] K. Zhang, J. Lu, G. Lafruit, R. Lauwereins, and L. Van Gool, "Robust stereo matching with fast normalized cross-correlation over shape-adaptive regions," in Proceedings of the IEEE International Conference on Image Processing (ICIP' 09), pp. 2357-2360, Cairo, Egypt, November 2009.

[19] X.-L. Xiong, The Image Segmentation Algorithm Based on Texture Gradient, Hefei University of Technology, Hefei, China, 2012.

[20] Z.-M. Li, Research on automatic tree generation algorithm based on L system [M.S. thesis], Huazhong University of Science and Technology, Wuhan, China, 2011.

[21] I. Shlyakhter, M. Rozenoer, J. Dorsey, and S. Teller, "Reconstructing 3D tree models from instrumented photographs," IEEE Computer Graphics and Applications, vol. 21, no. 3, pp. 5361, 2001.

[22] C. Xu, D. Zhang, Z. Zhang, and Z. Feng, "BgCut: automatic ship detection from UAV images," The Scientific World Journal, vol. 2014, Article ID 171978, 11 pages, 2014.

[23] L. Vincent and P. Soille, "Watersheds in digital spaces: an efficient algorithm based on immersion simulations," IEEE Transactions on Pattern Analysis and Machine Intelligence, vol. 13, no. 6, pp. 583-598, 1991.

[24] D. G. Lowe, "Distinctive image features from scale-invariant keypoints," International Journal of Computer Vision, vol. 60, no. 2, pp. 91-110, 2004.

[25] A. Lindenmayer and D. Frijters, "A model for the growth and flowering of aster novae-angliae on the basis of table $<1 ; 0>\mathrm{L}$ systems," in L Systems, G. Rozenberg and A. Salomaa, Eds., vol. 15 of Lecture Notes in Computer Science, pp. 24-52, Springer, Berlin, Germany, 1974. 


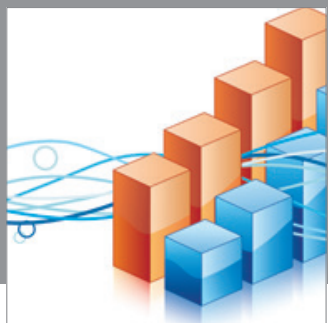

Advances in

Operations Research

mansans

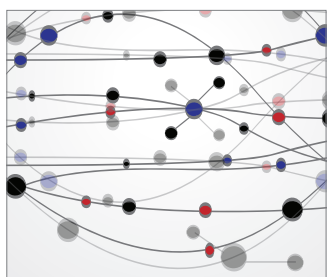

The Scientific World Journal
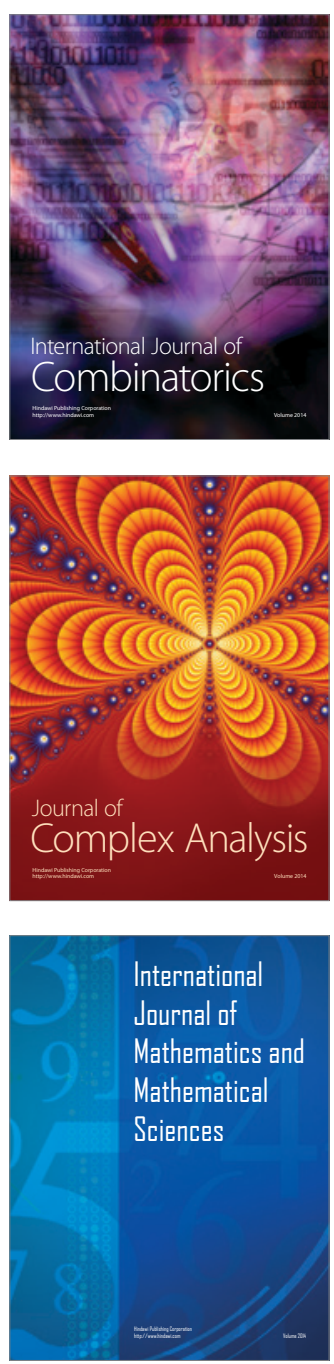
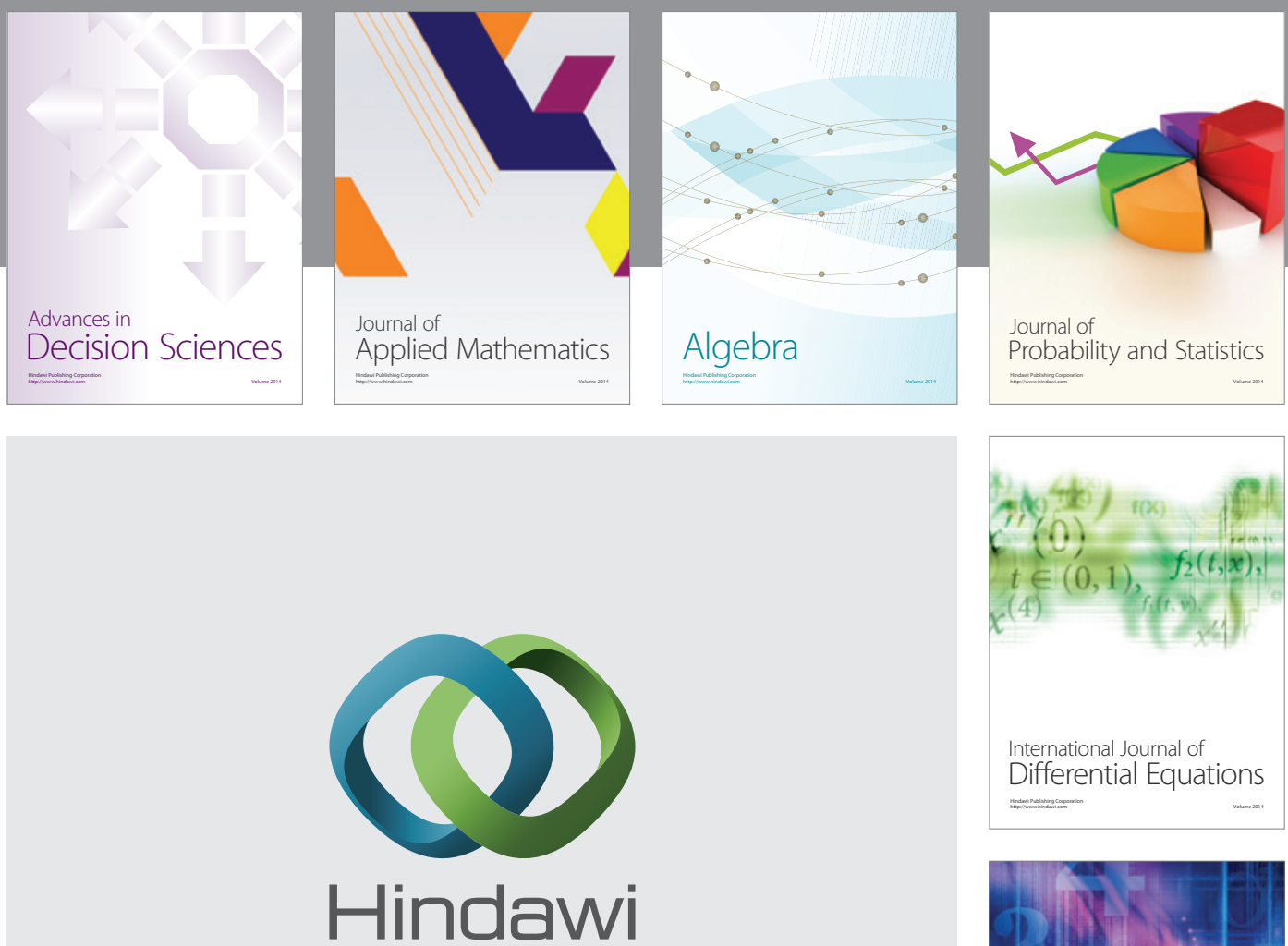

Submit your manuscripts at http://www.hindawi.com
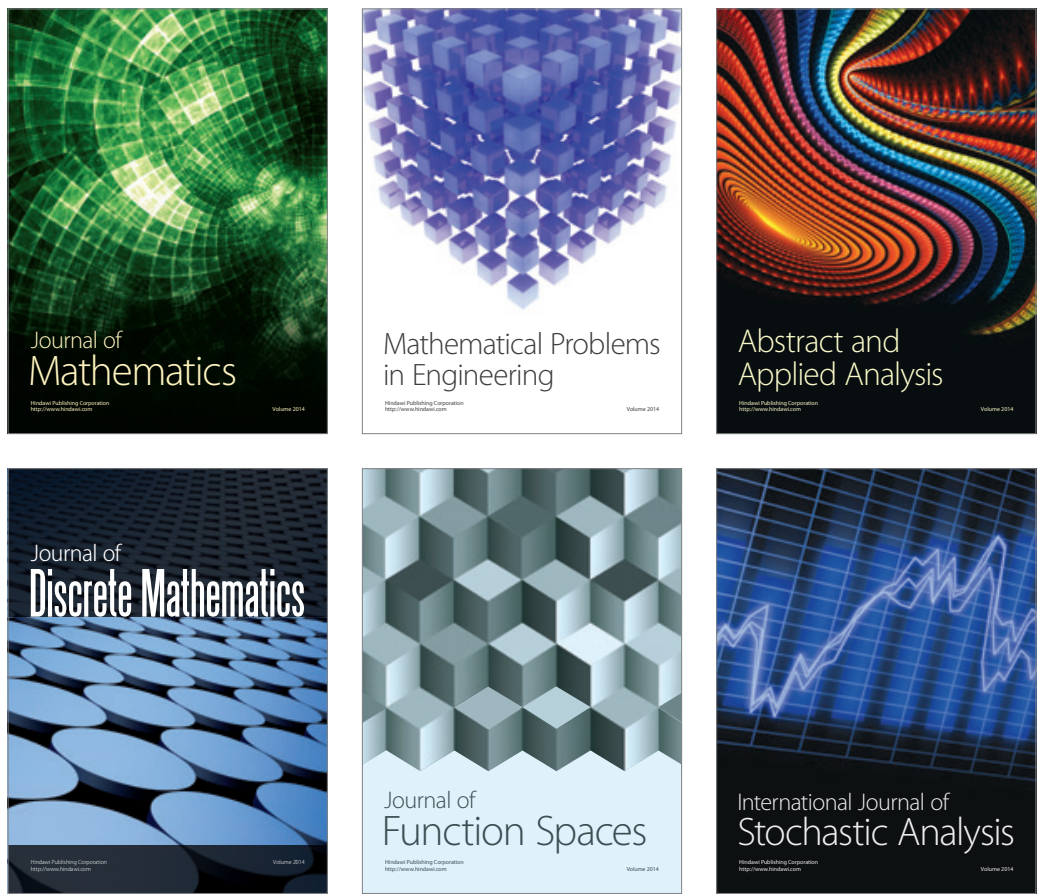

Journal of

Function Spaces

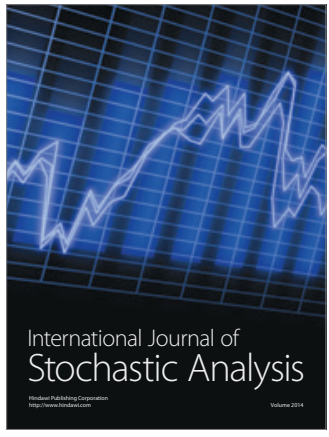

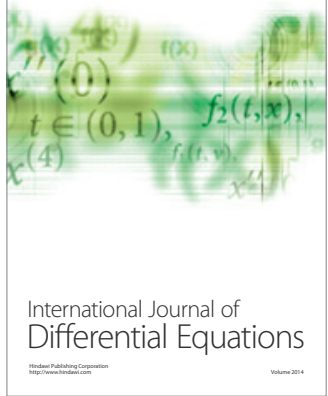
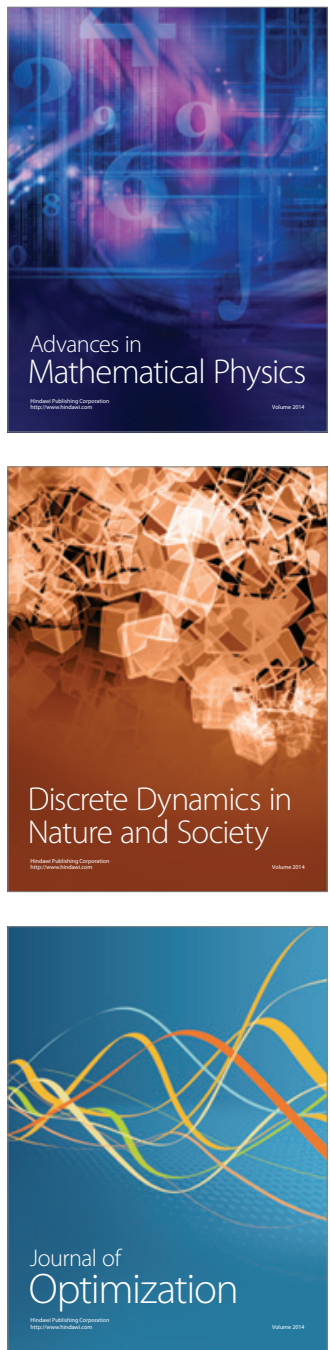